\title{
Loop-Mediated Isothermal Amplification-Coupled Glass Nanopore Counting Towards Sensitive and Specific Nucleic Acid Testing
}

Zifan Tang, ${ }^{\dagger}$ Gihoon Choi ${ }^{\dagger}$ Reza Nouri, $^{\dagger}$ and Weihua Guan ${ }^{\dagger}, \star^{*}$

$\dagger$ Department of Electrical Engineering, Pennsylvania State University, University Park, Pennsylvania 16802, United States

$\$$ Department of Biomedical Engineering, Pennsylvania State University, University Park, Pennsylvania 16802, United States

* Corresponding author: W. Guan (wzg111@psu.edu)

This PDF file includes:

- Notes S1 to S3

- Figures S1 to S4

- Tables S1 to S4

- Supplementary References 


\section{Note S1. Mixed-use of capture rate and translocation rate}

Note that there were mixed usages of the term 'capture rate' and 'translocation rate' in literature. For example, the term 'translocation rate' could refer to (1) the time it takes for the molecule to pass through the pore area (unit: s) ${ }^{1-2}$, or (2) event rate of molecules going through the pore (unit: $\left.\mathrm{s}^{-1}\right)^{3}$, or $(3)$ concentration normalized event rate (unit: $\mathrm{s}^{-1} \mathrm{M}^{-1}$ ) ${ }^{4-5}$. The term 'capture rate' could refer to (1) event rate of molecules going through the pore (unit: $\mathrm{s}^{-1}$ ) ${ }^{1,6-7}$ or (2) concentration normalized event rate (unit: $\mathrm{s}^{-1} \mathrm{M}^{-1}$ ) ${ }^{3,8}$. To avoid confusion, we used the term 'event rate' in this work to refer to the rate that consecutive molecules go through the nanopore.

\section{Note S2. Average molecular distance estimation}

In a 1 Molar solution, there are $6 \times 10^{23}$ molecules per liter of liquid, or 0.6 molecules per $\mathrm{nm}^{3}$. Therefore, the volume per molecule is $1.66 \mathrm{~nm}^{3}$ at $1 \mathrm{M}$. For any molar concentration $C$, the volume per molecule is $1.66 \mathrm{~nm}^{3} / \mathrm{C}$. Take the cubic root of this volume per molecule, and we can obtain the average separation of molecules as $d=1.18 / C^{1 / 3}$, where $C$ is in molar and $d$ is in nanometer. The average molecular distance at $12 \mathrm{pM}$ is thus estimated to be $5.2 \mu \mathrm{m}$. Similarly, the average molecular distance at $60 \mathrm{pM}$ is estimated to be $3 \mu \mathrm{m}$.

\section{Note S3. Fitting event rate versus amplification time with the logistic growth model}

LAMP reaction is an auto-cycling process which involves multiple pathways for gene replication, and this reaction amplifies the DNA from the single copy to a billion copies in less an hour ${ }^{9}$. At the early phase, the number of amplicons exponentially increases with time. However, as the reaction goes, it starts to be limited by remaining reagents in the master reaction mix (dNTPs, primers, and enzymes). The rate of amplicon synthesis will eventually cease. The growth rate as the function of time can be express as ${ }^{10}$

$$
\frac{d N(t)}{d t}=\beta N(t)(\Omega-(N(t))
$$

in which $N(t)$ is the number of the amplicons at time $t, b$ is the constant rate of growth, $\Omega$ is the saturation level of amplicon numbers. This means the growth rate is affected by both the existing amplicon numbers $N(t)$ and the remaining resources $\Omega-N(t)$. The solution to Eq. (S1) can be written as a 4 parameter logistic function ${ }^{11-12}$ 
Supplementary Information

$$
N(t)=A+\frac{\Omega-A}{1+e^{-\beta\left(t-t_{0}\right)}}
$$

in which $A$ is the lower bound of the amplicon numbers, $t_{0}$ is the time at the maximum growth rate.

In the nanopore digital counting, the event rate $R$ has a linear relationship with the concentration as

$$
R=\alpha N(t) / V_{\text {Reaction }}
$$

where $\alpha=2 \pi D r^{*}$, and $r^{*}$ is the capture radius which contains information about the molecular diffusion coefficient, the nanopore geometry and the experimentally applied voltage ${ }^{13}$, and $V_{\text {Reaction }}$ (with unit $\mathrm{m}^{3}$ ) is the LAMP mixture volume, which is $25 \mu \mathrm{l}$ in our experiment.

Combining Eq. S2 and Eq. S3, the event rate can be written as:

$$
\mathrm{R}(t)=R_{L}+\frac{R_{H}-R_{L}}{1+e^{-\beta\left(t-t_{0}\right)}}
$$

in which $R_{L}=\alpha A / V_{\text {Reaction }}$ is the lower bound of the event rate. $R_{H}=\alpha \Omega / V_{\text {Reaction }}$ is the saturation level of the event rate. Eq. S4 combines both the nanopore characteristics and the LAMP amplification dynamics and is used for fitting the amplification curve in nanopore counting. 

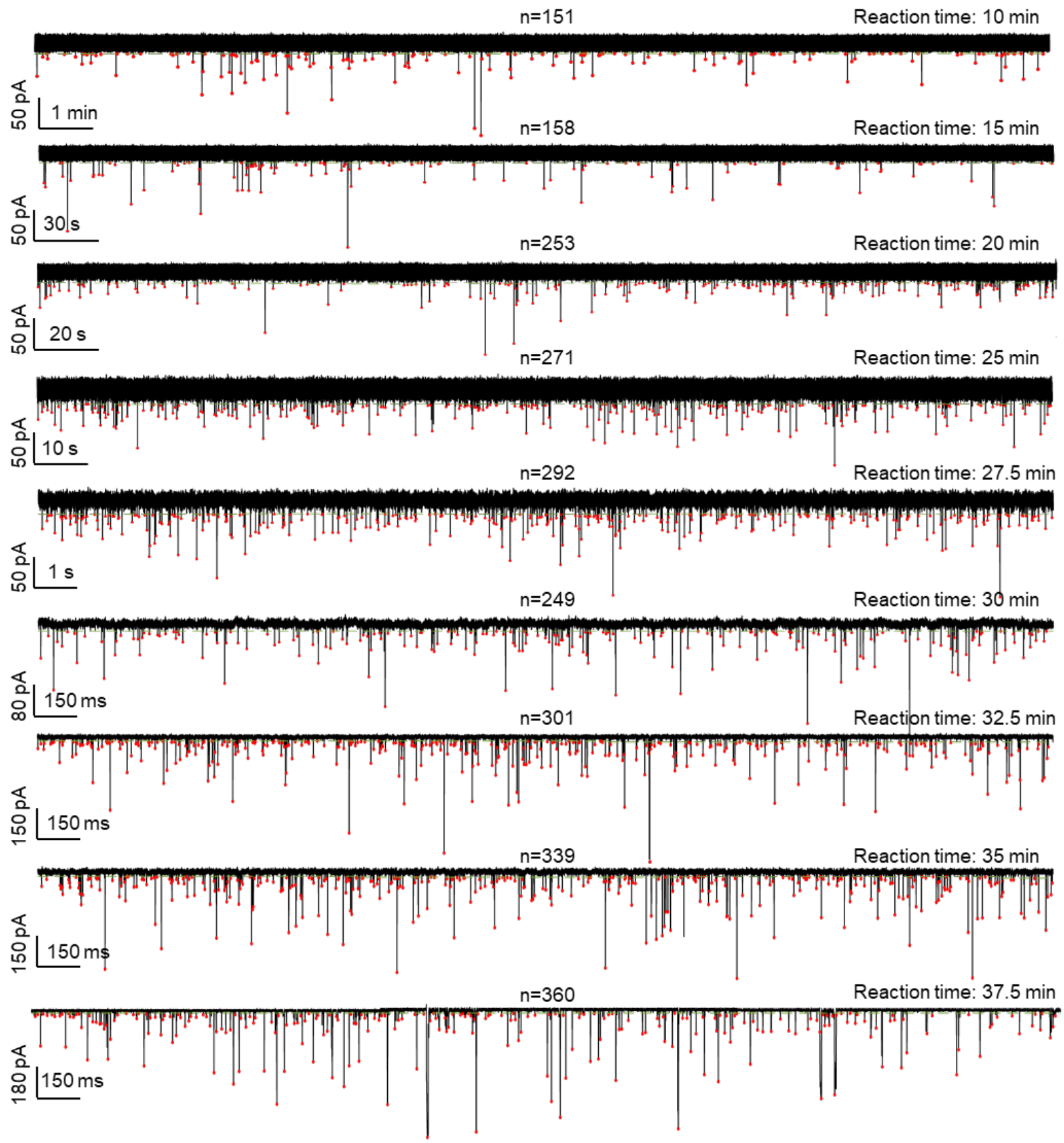

Figure S1. Summary of all current time traces obtained after indicated LAMP reaction time (the Pf genomic DNAs concentration is the $100 \mathrm{ng} / \mu \mathrm{l})$. The green dash line is the noise threshold and the red dots represent the peak location of the current dip. ( $n$ : total number of translocation events detected). Note the differences in time scale. 
Supplementary Information
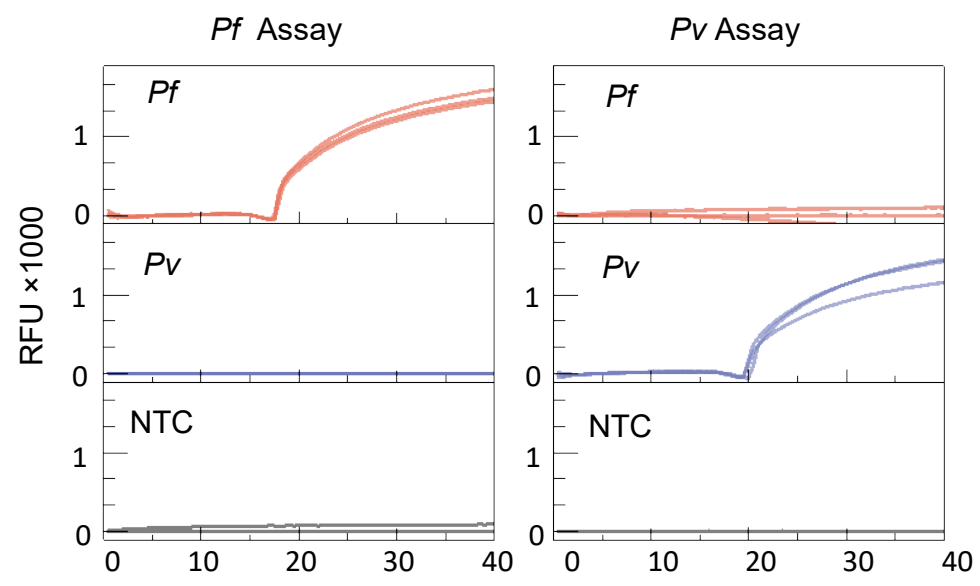

Figure S2. Validation of the $P f$ and $P v$ LAMP assays using $P f$ and $P v$ genomic DNA in benchtop real-time PCR machine (NTC: no template control).
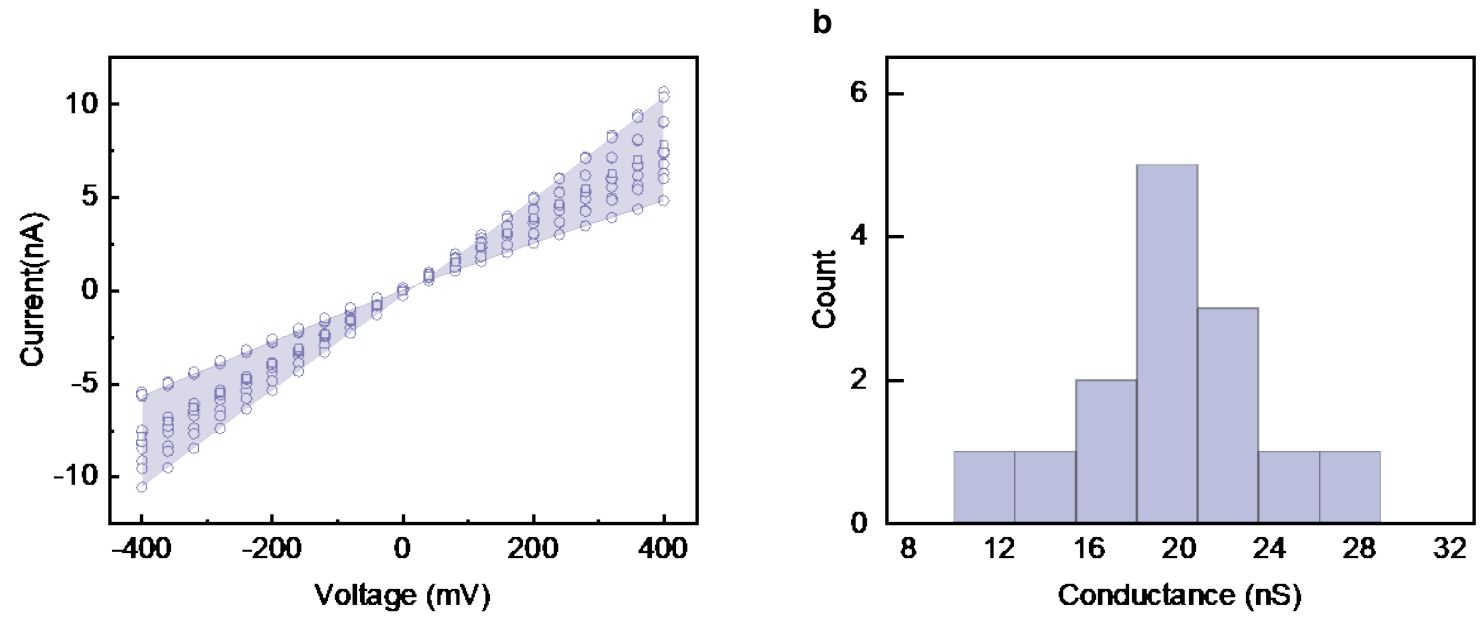

Figure S3. I-V characterization for all glass nanopore used. (a) Current-voltage curves for the glass nanopore in $1 \mathrm{M} \mathrm{KCl}$ with Tris-EDTA-buffer solution. (b) Conductance distribution of the glass nanopores. The nanopore size clearly varies from batch to batch. Typical conductance of the fabricated nanopore is in the range of $20 \pm 10 \mathrm{nS}$. 
Supplementary Information
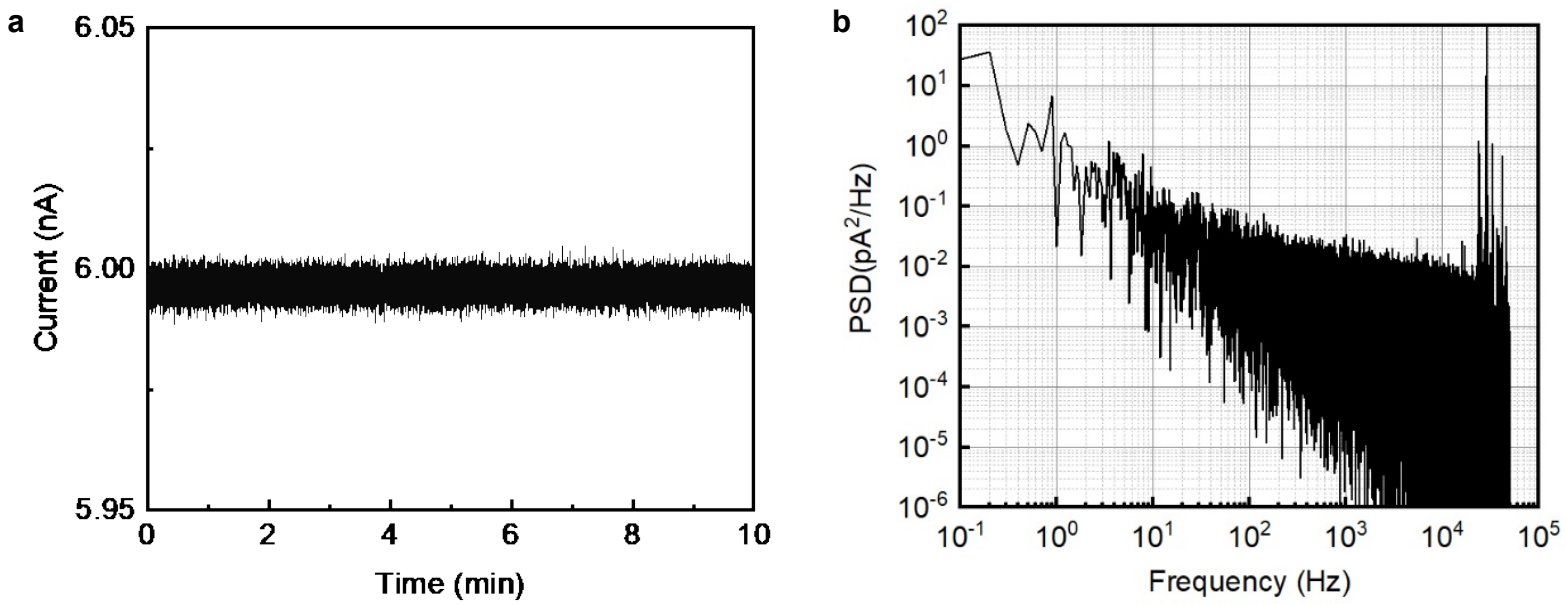

Figure S4. Typical noise performance of the glass nanopore sensor in $1 \mathrm{M} \mathrm{KCl}$ Tris-EDTA buffer at $400 \mathrm{mV}$. (a) Current traces, the RMS noise was around $4.2 \mathrm{pA}$, (b) Power spectrum density for the ionic current signal. 
Supplementary Information

Table S1. Extracted event rate after the specific LAMP reaction time

\begin{tabular}{llllllllllll}
\hline & $10 \min$ & $15 \min$ & $20 \min$ & $25 \min$ & $27.5 \min$ & $30 \min$ & $32.5 \min$ & $35 \min$ & $37.5 \min$ & $40 \mathrm{~min}$ & $42.5 \min$ \\
\hline $1 \mathrm{X}$ & $0.1 / \mathrm{s}$ & $0.20 / \mathrm{s}$ & $0.38 / \mathrm{s}$ & $2.09 / \mathrm{s}$ & $14.6 / \mathrm{s}$ & $83.0 / \mathrm{s}$ & $112 / \mathrm{s}$ & $112 / \mathrm{s}$ & $120 / \mathrm{s}$ & NA & NA \\
$0.1 \mathrm{X}$ & NA & NA & $0.16 / \mathrm{s}$ & $0.47 / \mathrm{s}$ & $1.55 / \mathrm{s}$ & $4.22 / \mathrm{s}$ & $13.8 / \mathrm{s}$ & $36.3 / \mathrm{s}$ & $120 / \mathrm{s}$ & $130 / \mathrm{s}$ & $\mathrm{NA}$ \\
$0.01 \mathrm{X}$ & $\mathrm{NA}$ & $\mathrm{NA}$ & $\mathrm{NA}$ & $\mathrm{NA}$ & $0.17 / \mathrm{s}$ & $0.40 / \mathrm{s}$ & $2.10 / \mathrm{s}$ & $9.60 / \mathrm{s}$ & $38.0 / \mathrm{s}$ & $54.5 / \mathrm{s}$ & $117 / \mathrm{s}$ \\
\hline
\end{tabular}

Table S2. Parameter values for fitting the logistic function fit in Figure 7

\begin{tabular}{cccccc}
\hline & $R_{L}\left(s^{-1}\right)$ & $R_{H}\left(s^{-1}\right)$ & $\beta\left(\mathrm{min}^{-1}\right)$ & $t_{0}($ min $)$ & Fitting $R^{2}$ \\
\hline $1 \mathrm{X}$ & 0.1 & 123.2 & 0.75 & 29.2 & 0.99 \\
$0.1 \mathrm{X}$ & 0.1 & 123.2 & 0.75 & 35.3 & 0.97 \\
$0.01 \mathrm{X}$ & 0.1 & 123.2 & 0.75 & 39.8 & 0.95 \\
\hline
\end{tabular}

Table S3. Reagent setup of LAMP master mix

\begin{tabular}{lll}
\hline Component & Concentration & Volume \\
\hline PCR grade water & $1 \mathrm{x}$ & $9.25 \mu \mathrm{l}$ \\
Primer sets & - & $6.50 \mu \mathrm{l}$ \\
Isothermal buffer & $1 \mathrm{x}$ & $2.50 \mu \mathrm{l}$ \\
$\mathrm{MgSO}_{4}$ & $7.00 \mathrm{mM}$ & $1.75 \mu \mathrm{l}$ \\
$\mathrm{Calcein}$ & $25.00 \mu \mathrm{M}$ & $0.63 \mu \mathrm{l}$ \\
$\mathrm{MnCl}_{2}$ & $0.75 \mathrm{mM}$ & $1.88 \mu \mathrm{l}$ \\
dNTP mix $_{\text {Bst } \text { DNA polymerase }}$ & $1.40 \mathrm{mM}$ & $3.50 \mu \mathrm{U}$ \\
\hline
\end{tabular}

Table S4. Primer sets for $P f$-, and $P v$-specific LAMP amplification.

\begin{tabular}{|c|c|c|}
\hline Species & Primer & Sequence $(5$ ' $\rightarrow$ 3') \\
\hline \multirow[t]{6}{*}{ P. falciparum ${ }^{14}$} & F3 & CTCCATGTCGTCTCATCGC \\
\hline & $\mathrm{B} 3 \mathrm{c}$ & AACATTTTTTAGTCCCATGCTAA \\
\hline & FIP (F1c - F2) & ACCCAGTATATTGATATTGCGTGACAGCCTTGCAATAAATAATATCTAGC \\
\hline & $\mathrm{BIP}(\mathrm{B} 1-\mathrm{B} 2 \mathrm{c})$ & AACTCCAGGCGTTAACCTGTAATGATCTTTACGTTAAGGGC \\
\hline & LF & CGGTGTGTACAAGGCAACAA \\
\hline & LB & GTTGAGATGGAAACAGCCGG \\
\hline \multirow[t]{6}{*}{ P. vivax 15} & F3 & GGTACTGGATGGACTTTATAT \\
\hline & $\mathrm{B} 3 \mathrm{c}$ & GGTAATGTTAATAATAGCATTACAG \\
\hline & FIP (F1c - F2) & CCAGATACTAAAAGACCAACCCACCATTAAGTACATCACT \\
\hline & $\mathrm{BIP}(\mathrm{B} 1-\mathrm{B} 2 \mathrm{c})$ & GCTAGTATTATGTCTTCTTTCACTTAATATACCAAGTGTTAAACC \\
\hline & LF & GATAACATCTACTGCAACAGG \\
\hline & LB & CTACTGTAATGCATCTAAGATC \\
\hline
\end{tabular}




\section{Supplementary References}

S1. Meller, A.; Branton, D., Single molecule measurements of DNA transport through a nanopore. Electrophoresis 2002, 23 (16), 2583-2591.

S2. Wanunu, M.; Sutin, J.; McNally, B.; Chow, A.; Meller, A., DNA translocation governed by interactions with solid-state nanopores. Biophysical journal 2008, 95 (10), 4716-4725.

S3. Freedman, K. J.; Haq, S. R.; Fletcher, M. R.; Foley, J. P.; Jemth, P.; Edel, J. B.; Kim, M. J., Nonequilibrium capture rates induce protein accumulation and enhanced adsorption to solid-state nanopores. ACS nano 2014, 8 (12), 12238-12249.

S4. Bell, N. A.; Muthukumar, M.; Keyser, U. F., Translocation frequency of double-stranded DNA through a solid-state nanopore. Physical review. E 2016, 93 (2), 022401.

S5. Ermann, N.; Hanikel, N.; Wang, V.; Chen, K. K.; Weckman, N. E.; Keyser, U. F., Promoting singlefile DNA translocations through nanopores using electro-osmotic flow. Journal of Chemical Physics 2018, 149 (16), 163311.

S6. Zhang, J.; Shklovskii, B. I., Effective charge and free energy of DNA inside an ion channel. Physical review. E, Statistical, nonlinear, and soft matter physics 2007, 75 (2 Pt 1), 021906.

S7. Freedman, K. J.; Otto, L. M.; Ivanov, A. P.; Barik, A.; Oh, S.-H.; Edel, J. B., Nanopore sensing at ultra-low concentrations using single-molecule dielectrophoretic trapping. Nature communications 2016, 7, 10217.

S8. Grosberg, A. Y.; Rabin, Y., DNA capture into a nanopore: interplay of diffusion and electrohydrodynamics. The Journal of chemical physics 2010, 133 (16), 165102.

S9. Notomi, T.; Okayama, H.; Masubuchi, H.; Yonekawa, T.; Watanabe, K.; Amino, N.; Hase, T., Loopmediated isothermal amplification of DNA. Nucleic acids research 2000, 28 (12), e63-e63.

S10. Cramer, J. S., The early origins of the logit model. Studies in History and Philosophy of Science Part C: Studies in History and Philosophy of Biological and Biomedical Sciences 2004, 35 (4), 613-626.

S11. Fekedulegn, D.; Mac Siurtain, M. P.; Colbert, J. J., Parameter estimation of nonlinear growth models in forestry. Silva Fennica 1999, 33 (4), 327-336.

S12. Subramanian, S.; Gomez, R. D., An empirical approach for quantifying loop-mediated isothermal amplification (LAMP) using Escherichia coli as a Model System. PloS one 2014, 9 (6), e100596.

S13. Grosberg, A. Y.; Rabin, Y., DNA capture into a nanopore: interplay of diffusion and electrohydrodynamics. The Journal of chemical physics 2010, 133 (16), $10 \mathrm{~B} 617$.

S14. Polley, S. D.; Mori, Y.; Watson, J.; Perkins, M. D.; Gonzalez, I. J.; Notomi, T.; Chiodini, P. L.; Sutherland, C. J., Mitochondrial DNA targets increase sensitivity of malaria detection using loopmediated isothermal amplification. J Clin Microbiol 2010, 48 (8), 2866-71.

S15. Britton, S.; Cheng, Q.; Grigg, M. J.; Poole, C. B.; Pasay, C.; William, T.; Fornace, K.; Anstey, N. M.; Sutherland, C. J.; Drakeley, C., Sensitive detection of Plasmodium vivax using a highthroughput, colourimetric loop mediated isothermal amplification (HtLAMP) platform: a potential novel tool for malaria elimination. PLoS neglected tropical diseases 2016, 10 (2), e0004443. 